\title{
Thermodynamic properties of neutral particle in the presence of topological defects in magnetic cosmic string background
}

\author{
H. Hassanabadi, M. Hosseinpour ${ }^{\mathrm{a}}$ \\ Physics Department, Shahrood University of Technology, Shahrood, Iran
}

Received: 12 April 2016 / Accepted: 19 September 2016 / Published online: 8 October 2016

(c) The Author(s) 2016. This article is published with open access at Springerlink.com

\begin{abstract}
In this paper, we study the covariant form of the non-relativistic Schrödinger-Pauli equation in the spacetime generated by a cosmic string and discuss the solutions of this equation in the presence of interaction between the magnetic dipole momentum and electromagnetic field. We study the influence of the topology on this system. We obtain the solution of radial part as well as the energy levels. We consider all thermodynamic properties of a neutral particle in a magnetic cosmic string background by using an approach based on the partition function method.
\end{abstract}

\section{Introduction}

The interaction between electric and magnetic fields and multipole moments has attracted attention in a great deal of studies, such as [1-13], the holonomic quantum computation [14-16], and the Landau quantization [17,18]. Besides, recent studies of the interaction between a moving electric moment and external fields [11,12] have shown a difference between the field configuration that yields the occurrence of geometric phases for an electric charge $[1,2,19]$, an electric dipole moment $[3,4,9,10]$, and a moving electric moment. The quantum mechanics dynamics on conical spaces in the presence of topological defects has attracted much attention in recent years [20-22]. Among different motivations we can recall the context of the $(2+1)$ dimensional quantum gravity [23] and cosmic strings [24]. The simple, but nontrivial, geometry of the cone appears as an effective geometry in such diverse physical entities as cosmic strings [25]. Accordingly, the dynamics of quantum neutral particles in a conical background has been studied with very different motivations [26-29]. An important issue concerning the cone is the fact that the conical background is naturally associated to a curvature singularity at the cone tip. The quantum dynamics of a single particle in a coni-

a e-mail: hosseinpour.mansoureh@gmail.com cal space-time has been investigated by several authors. Till now, some problems have been investigated in a conical space-time including classical and quantum non-relativistic dynamics of a particle [30] and the influence of conical singularities in the energy levels of a harmonic oscillator [31].

In our work, we are going to discuss the solution of the non-relativistic Schrödinger-Pauli equation produced by a cosmic string. The latter is a linear defect that changes the topology of the medium. Topological defects will arise in some of the models with spontaneous symmetry breakdown in field theory. The cosmic strings are expected to have large mass density and very thin width. The spacetime geometry around an infinitely stretching straight string has a peculiar property. From the field theory point of view, the cosmic string can be viewed as a consequence of a symmetry breaking phase transition in the early universe [32]. Till now, some problems have been investigated in curved space-time including the one-electron atom

This paper is organized as follows. In Sect. 2, we first review the covariant non-relativistic Schrödinger-Pauli equation in the space-time generated by a cosmic string. We next report the solution of the radial part as well as the energy levels. Finally, in Sect. 3, We consider all thermodynamic properties of neutral particle in magnetic cosmic string background by using an approach based on the partition function method.

\section{Neutral particle in cosmic string background}

The cosmic string space-time with an internal magnetic field in cylindrical coordinates is described by the line element [33-36]

$\mathrm{d} s^{2}=-\mathrm{d} t^{2}+\mathrm{d} r^{2}+\alpha^{2} r^{2} \mathrm{~d} \phi^{2}+\mathrm{d} z^{2}$ 
with $-\infty<z<\infty, r \geq 0$, and $0 \leq \varphi \leq 2 \pi$. The parameter $\alpha$ is related to the linear mass density $\tilde{m}$ of the string via $\alpha=1-4 \tilde{m}$ and varies in the interval $(0,1]$.

We can build the local reference frame through a noncoordinate basis with $e_{\mu}^{(a)}$ where $e_{\mu}^{(a)}$ and $e_{(a)}^{\mu}(x)$ are transformation matrices. The components of the non-coordinate basis $e_{\mu}^{(a)}$ are called tetrads or vierbeins that form our local reference frame and $e_{(a)}^{\mu}(x)$ satisfy

$\eta^{a b} e_{\bar{a}}^{\mu}(x) e_{\bar{b}}^{v}(x)=g^{\mu \nu}(x)$

where $\mu, v=0,1,2,3$ are tensor indices and $\bar{a}, \bar{b}=$ $0,1,2,3$ denote tetrad indices $[37,38]$. We can obtain the component of spin connection from

$\omega_{\mu \bar{b}}^{\bar{a}}=e_{\nu}^{\bar{a}} e_{\bar{b}}^{\sigma} \Gamma_{\sigma \mu}^{\nu}+e_{\nu}^{\bar{a}} \partial_{\mu} e_{\bar{b}}^{\nu}$

$\Gamma_{\mu \nu}^{\sigma}$ are the Christoffel symbols of the second kind. The nonvanishing components of the spin connection are

$\omega_{\varphi}^{\overline{12}}=e_{\mu}^{\overline{1}} e^{\nu \overline{2}} \Gamma_{\varphi \nu}^{\mu}-e^{\nu \overline{2}} \partial_{\varphi} e_{\mu}^{\overline{1}}==1-\alpha$,

$\omega_{\varphi}^{1 \overline{12}}=-\omega_{\varphi}^{\overline{2} \overline{1}}$.

The relativistic dynamics of the neutral particle in this curved space-time was studied in [39]. In the same paper, the non-relativistic behavior of the neutral particle in curved space-time was obtained through the application of the Foldy-Wouthuysen approximation [40] to the Dirac equation. We assume that the dipole magnetic moments are parallel to the $z$-axis of the space-time. The non-relativistic equation is [13]

$i \frac{\partial \psi}{\partial t}=m \psi+\left[\frac{1}{2 m} \vec{\pi}^{2}-\frac{\mu^{2} E^{2}}{2 m}+\frac{\mu}{2 m} \vec{\nabla} \cdot \vec{E}+\mu \hat{n} \cdot \vec{B}\right] \psi$

The unit vector $\hat{n}$ indicates the direction of the magnetic dipole moment. We introduce the generalized momentum in the presence of an electromagnetic field by

$\vec{\pi}=-i \hbar \vec{\nabla}+\left(\mu(\hat{n} \times \vec{E})+\frac{(1-\alpha)}{2} \hat{\varphi}\right)_{j}$

where

$\vec{\nabla}=\frac{\partial}{\partial r} \hat{r}+\frac{1}{\alpha r} \frac{\partial}{\partial \varphi} \hat{\varphi}+\frac{\partial}{\partial z} \hat{z}$

We choose the electric field as

$\vec{E}=\frac{\lambda \rho}{2} \hat{\rho}$ $\lambda$ is a linear density charge. By substituting Eq. (5) and by using the $\Psi=e^{-i E t} \psi$ we have

$$
\begin{aligned}
& -\frac{1}{2 m}\left(\frac{\partial^{2} \psi}{\partial \rho^{2}}+\frac{1}{\rho} \frac{\partial \psi}{\partial \rho}+\frac{1}{\alpha^{2} \rho^{2}} \frac{\partial^{2} \psi}{\partial \varphi^{2}}+\frac{\partial^{2} \psi}{\partial z^{2}}\right) \\
& -\frac{i}{2 m}\left(\frac{\mu \lambda}{\alpha}+\frac{(1-\alpha)}{\alpha^{2} \rho^{2}}\right) \frac{\partial \psi}{\partial \varphi}+\frac{\mu^{2} \lambda^{2}}{8 m} \rho^{2} \psi \\
& +\frac{1}{8 m} \frac{\left((1-\alpha)^{2}\right)}{\alpha^{2} \rho^{2}} \psi \frac{\mu \lambda}{2 m} \psi+\frac{\mu \lambda}{4 m} \frac{(1-\alpha)}{\alpha} \psi=E \psi .
\end{aligned}
$$

The solution of the Schrödinger-Pauli equation can be written in the form [13]

$\psi_{n l}(\rho, \varphi, z)=e^{i l \varphi} e^{i k z} R_{n l}(\rho)$

By substituting in Eq. (9)

$\left[-\frac{1}{2 m}\left(\partial_{\rho}^{2}+\frac{1}{\rho} \partial_{\rho}\right)+\frac{k^{2}}{2 m}+\frac{\gamma^{2}}{2 m \alpha^{2} \rho^{2}}+\frac{\gamma \omega}{2 \alpha}+\frac{m \omega^{2}}{8} \rho^{2}+\frac{\omega}{2}\right]$
$R_{n l}(\rho)=E R_{n l}(\rho)$

where we defined $\gamma=l+\frac{(1-\alpha)}{2}$ and $\omega=\mu \lambda / m$. We make the convenient change of variables of $\xi=\frac{m \omega}{2} \rho^{2}$.

Then we have [13]

$R_{n l}^{\prime \prime}+\frac{1}{\xi} R_{n l}^{\prime}+\frac{1}{\xi^{2}}\left(\xi \beta-\frac{\gamma^{2}}{4 \alpha^{2}}-\frac{\xi^{2}}{4}\right) R_{n l}=0$

where

$\beta=\frac{E}{\omega}-\frac{k^{2}}{2 m \omega}-\frac{\gamma}{2 \alpha}-\frac{1}{2}$

We can solve this equation. The corresponding wave functions and energy eigenvalues are obtained:

$\psi_{n l}=N \xi^{\frac{1}{2}+\sqrt{\frac{1}{4}+\frac{\gamma^{2}}{4 \eta^{2}}}} e^{-\frac{\xi}{2}} L_{n}^{1+2 \sqrt{\frac{1}{4}+\frac{\gamma^{2}}{4 \eta^{2}}}}(\xi)$,

$\varepsilon=\left(n+\frac{a}{2}\right) \omega$

where

$a=\left(2+\sqrt{1+\frac{\gamma^{2}}{\alpha^{2}}}+\frac{k^{2}}{m \omega}+\frac{\gamma}{\alpha}\right)$

and $N$ is the normalization constant. 
Fig. 1 The comparison of the Helmholtz free energy as a function of $K T$ for different angular quantum number $\psi$

Fig. 2 The comparison of the thermodynamic energy $U / N$ as a function of $K T$ for different angular quantum number
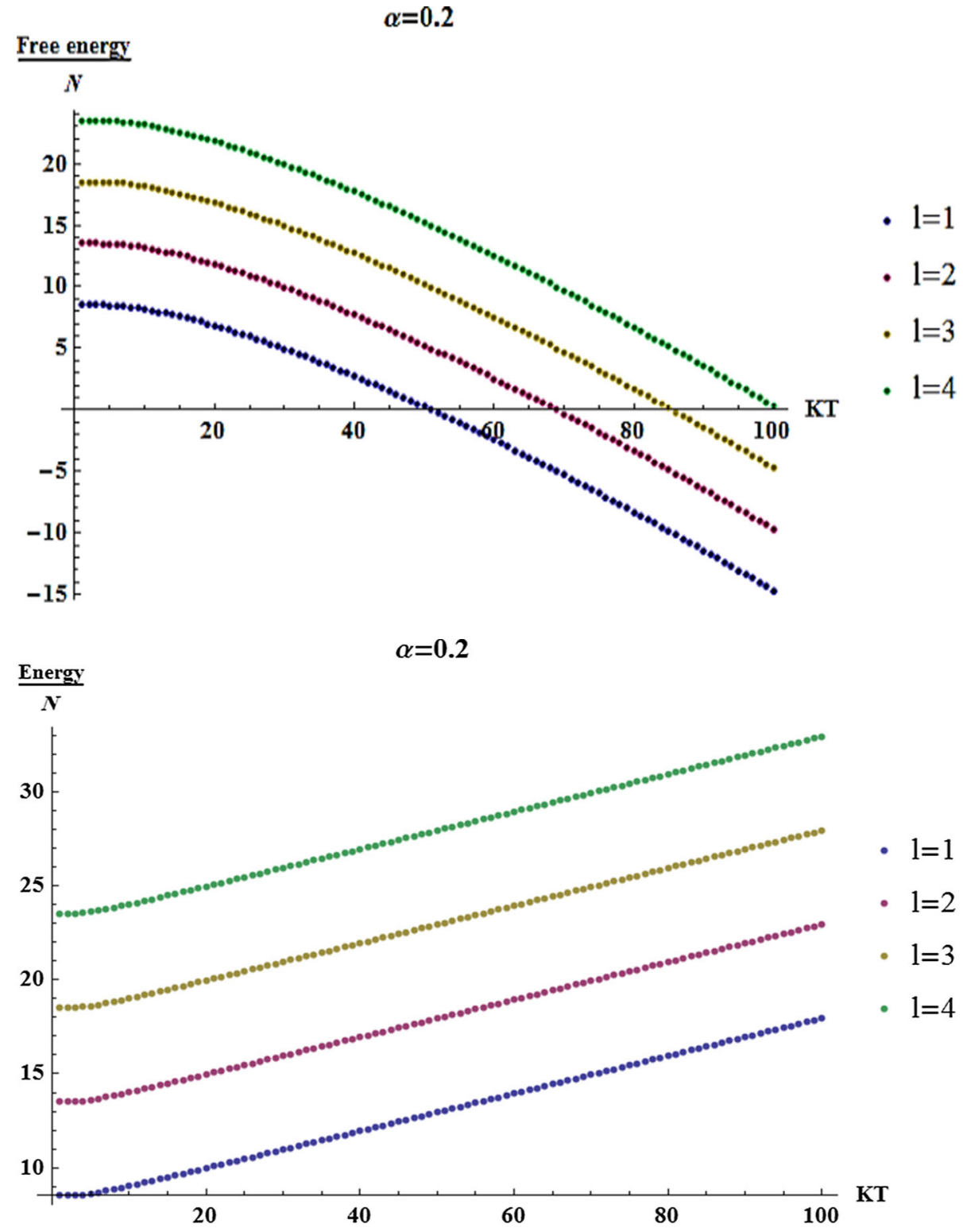

\section{Thermodynamic properties of system}

In order to consider thermodynamic properties of the neutral particle in a magnetic cosmic string background for a constant $\ell$ we concentrate, at first, on the calculation of the partition function,

$Q_{1}=\sum_{n=0}^{\infty} e^{-\beta\left(n+\frac{a}{2}\right) \omega}=e^{-\beta \omega \frac{(a-1)}{2}}\left\{2 \sinh \frac{\beta \omega}{2}\right\}^{-1}$,

where $\beta=1 / \kappa T$. The partition function for the N-body system with no interaction inside is obtained via

$Q_{N}=\left(Q_{1}\right)^{N}=e^{-N \beta \omega \frac{a}{2}}\left\{e^{\beta \omega}-1\right\}^{-N}$.
Once the Helmholtz free energy is obtained, the other statistical quantities are obtained in a straightforward manner,

$$
A=-\frac{1}{\beta} \ln Q_{N}=N \omega \frac{(a-1)}{2}+N K T \ln \left(2 \sinh \frac{\beta \omega}{2}\right) .
$$

The chemical potential can be obtained as

$\mu=\frac{\partial A}{\partial N}=\omega \frac{(a-1)}{2}+K T \ln \left(2 \sinh \frac{\beta \omega}{2}\right)$

and the pressure is zero,

$P=-\frac{\partial A}{\partial V}=0$. 
Fig. 3 The comparison of the Helmholtz free energy as a function of $K T$ for different $\alpha$

Fig. 4 The comparison of the thermodynamic energy $U / N$ as a function of $K T$ for different $\alpha$

Fig. 5 The entropy $S / N K$ as a function of $K T$ for different $\omega$
$1=3$
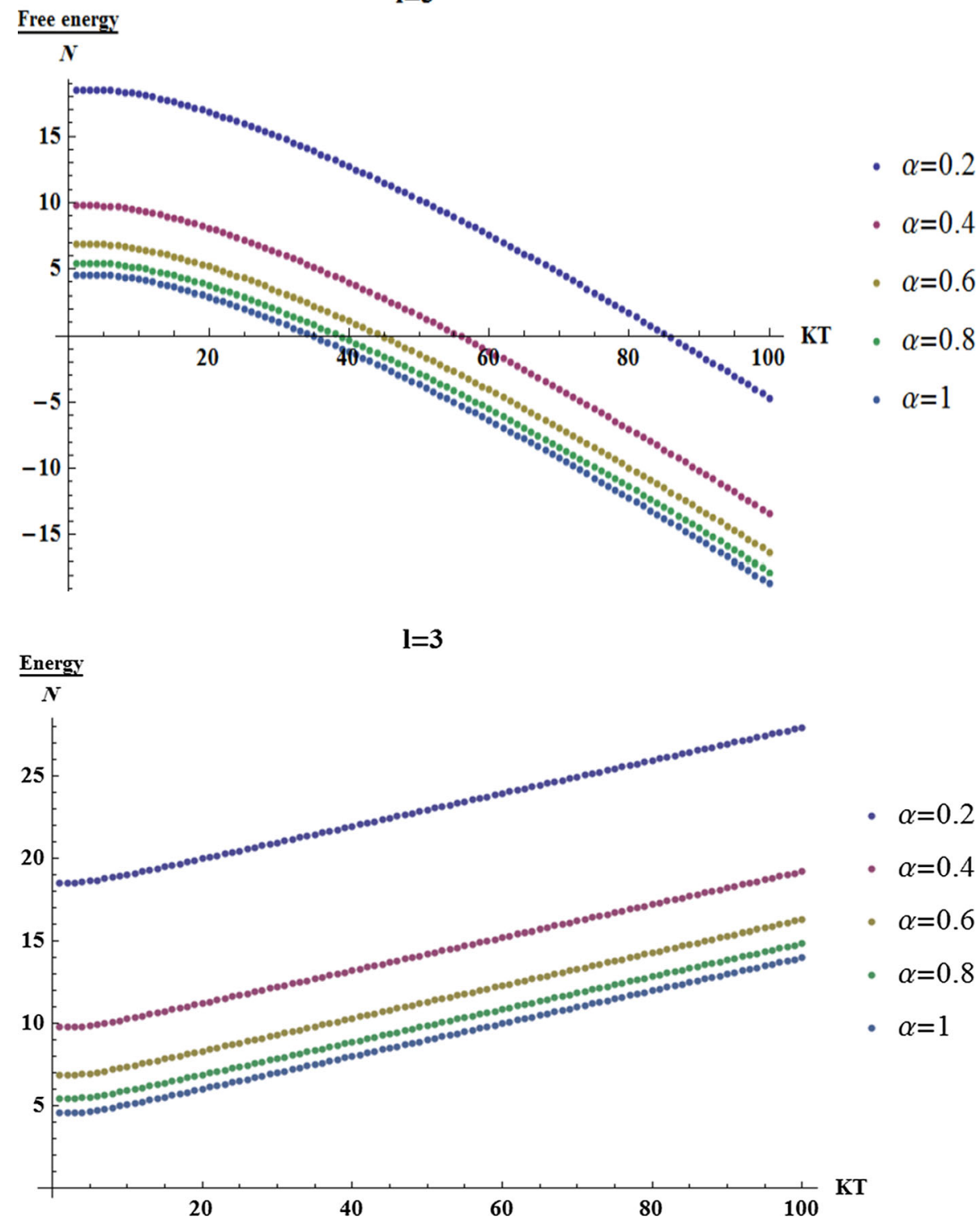

\section{Entropy}

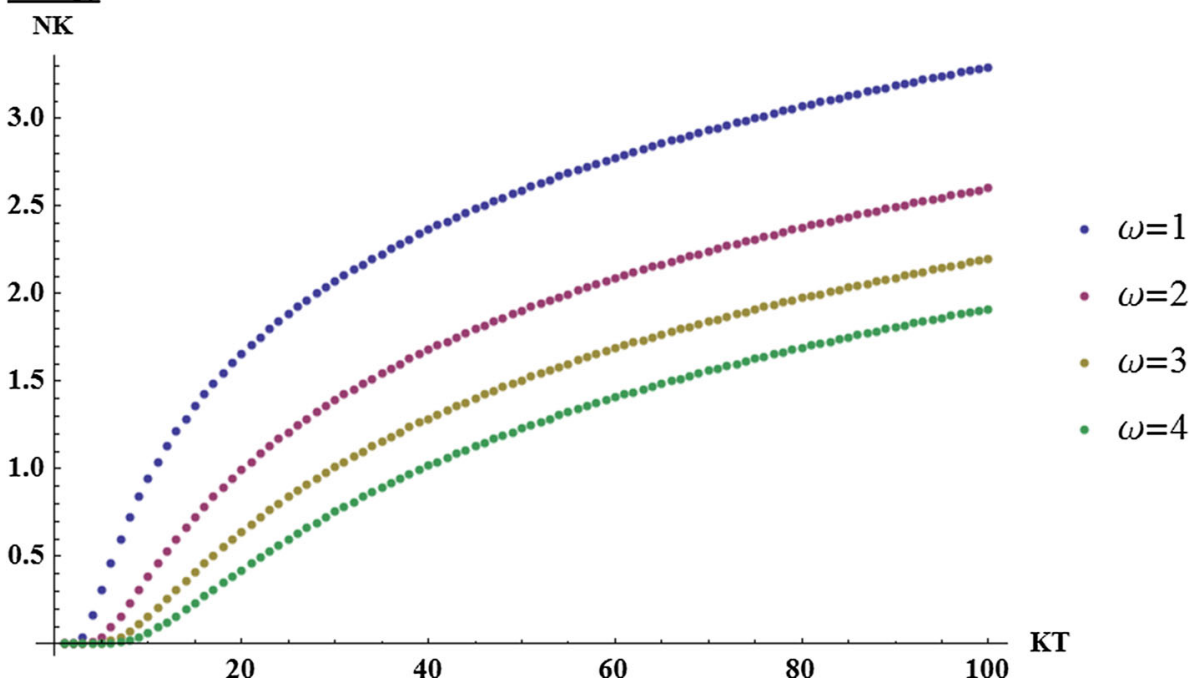


Fig. 6 The heat capacity $C / N K$ as a function of $K T$ for different $\omega$

Fig. 7 The entropy $S / N K$ as a function of $K T$ for $\omega=1$
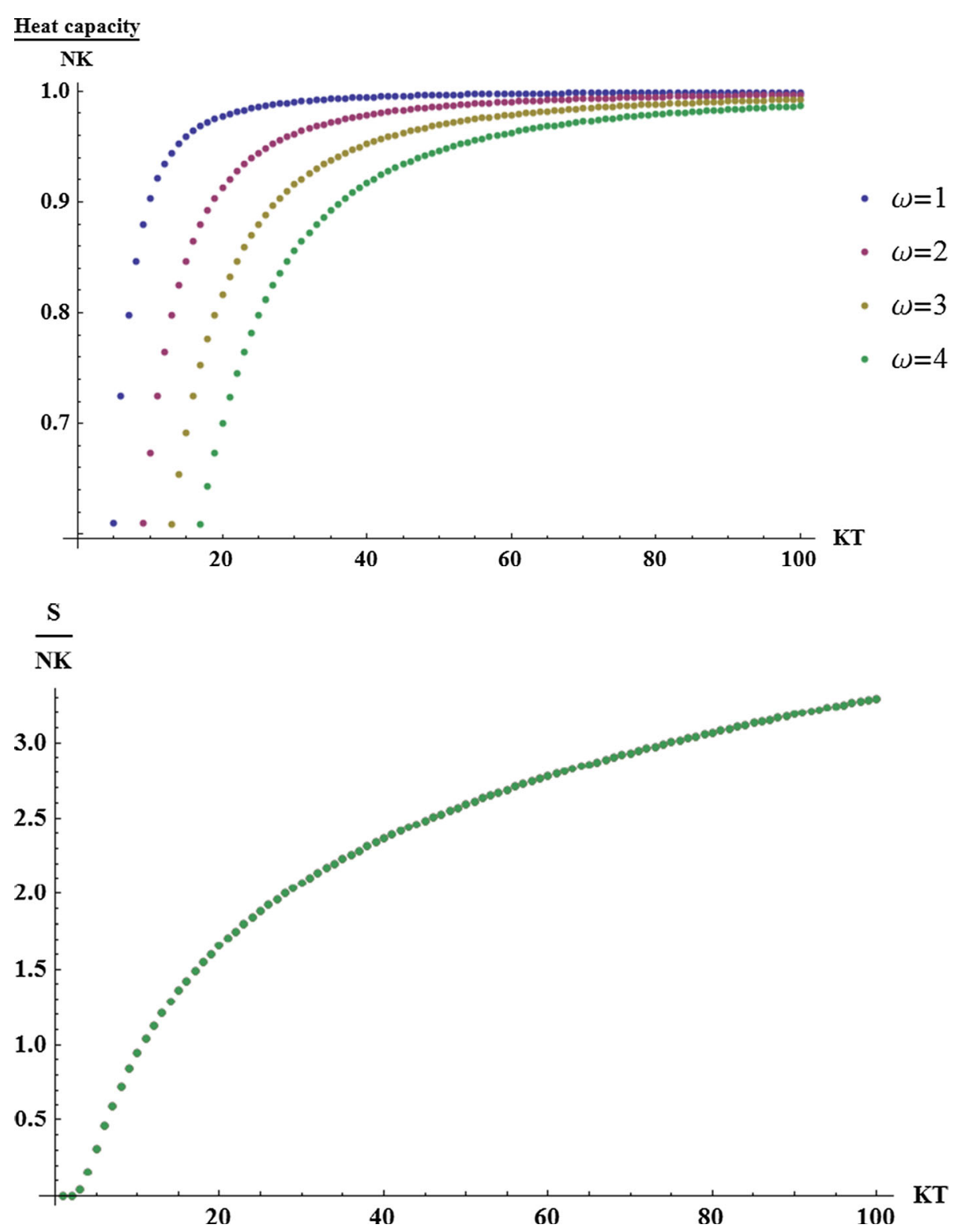

Once the Helmholtz free energy is obtained, the other statistical quantities are obtained in a straightforward manner.

The mean energy is

$U=-\frac{\partial \ln Q_{N}}{\partial \beta}=N \omega\left(\frac{a-1}{2}+\operatorname{coth} \frac{\beta \omega}{2}\right)$.

The main statistical quantity, i.e., the entropy, is related to the other quantities via

$\frac{S}{K}=\beta^{2} \frac{\partial A}{\partial \beta}=\left(-N \ln \left(2 \sinh \frac{\beta \omega}{2}\right)+N \beta \frac{\omega}{2} \operatorname{coth} \frac{\beta \omega}{2}\right) ;$ the specific heat capacity at constant volume is obtained from

$$
\frac{C}{K}=-\beta^{2} \frac{\partial U}{\partial \beta}=-\beta^{2} \omega^{2} N \frac{e^{-\beta \omega}}{1-e^{-\beta \omega}}
$$

One can verify that in the limit $\alpha \rightarrow 1$, the space-time becomes flat. We recover the general solution for flat spacetime in this limit where Eq. (12) is rewritten as

$R_{n l}^{\prime \prime}+\frac{1}{\xi} R_{n l}^{\prime}+\frac{1}{\xi^{2}}\left(\xi \beta-\frac{\gamma^{2}}{4}-\frac{\xi^{2}}{4}\right) R_{n l}=0$.

In this case $\gamma=l$ and

$\beta=\frac{E}{\omega}-\frac{k^{2}}{2 m \omega}-\frac{l}{2}-\frac{1}{2}$. 
Fig. 8 The heat capacity $C / N K$ as a function of $K T$ for $\omega=1$

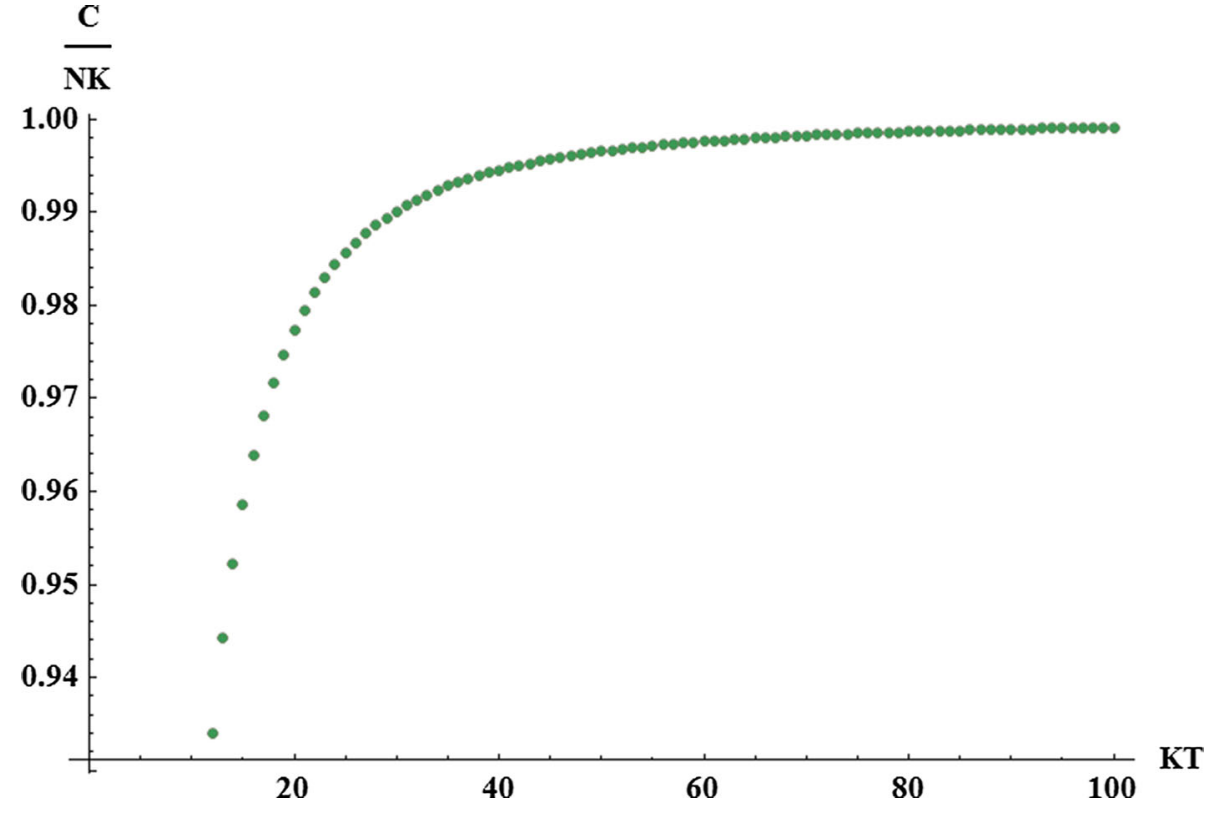

The corresponding wave functions and energy eigenvalues are obtained from the NU method as

$\psi=N^{\prime} \xi^{\frac{1}{2}\left(1+\sqrt{1+l^{2}}\right)} e^{-\frac{\xi}{2}} L_{n}^{1+\sqrt{1+l^{2}}}(\xi)$,

$\varepsilon=\left(n+\frac{a^{\prime}}{2}\right) \omega$,

where

$a^{\prime}=\left(2+\sqrt{1+l^{2}}+\frac{k^{2}}{m \omega}+l\right)$,

and $N^{\prime}$ is the normalization constant. In the limit $\alpha \rightarrow 1$ the thermodynamic properties of system will be obtained from Eqs. (17-24); the parameter $a$ must be replaced by $a^{\prime}$.

In the following we depict the thermodynamic properties of the system vs. $K T$ in Figs. 1, 2, 3, 4, 5, and 6. In Fig. 1 the Helmholtz free energy is plotted vs. $K T$ for different angular quantum number. In the interval $0 \leq K T \leq 100$, it is seen that the energy decreases with a nearly linear behavior for increasing $K T$. In Fig. 2, the thermodynamic energy is plotted vs. $K T$ for different angular quantum numbers in the interval $0 \leq K T \leq 100$. It reveals that, for increasing $K T$, the internal energy is linearly increased as well. As we see in Figs. 1 and 2 the Helmholtz free energy and thermodynamic energy both increase by increasing angular quantum number. Figure 3 shows the Helmholtz free energy plotted vs. $K T$ for different $\alpha$. In the interval $0 \leq K T \leq 100$, the energy decreases with a nearly linear behavior for increasing $K T$. Figure 4 represents the energy behavior vs. $K T$ for different $\alpha$ in the interval $0 \leq K T \leq 100$. As expected the thermodynamic properties tend to their behavior in flat spacetime when $\alpha$ tends to 1 . Figure 5 reveals that the entropy is slowly increasing for large $K T$ values. The curve is repeated for $\omega=1,2,3,4$. The variation of the heat capacity vs. $K T$ for various $\omega$ values is shown in Fig. 6. As can be seen in Figs. 5 and 6 , the entropy and the heat capacity decrease for increasing $\omega$. As we see in Figs. 7 and 8 the entropy and the heat capacity are independent of $\alpha$. As expected in Fig. 8 for increasing $K T$, the heat capacity tends to its saturation value in high temperature.

\section{Conclusion}

With the purpose of discussing the role of the topology on the Landau quantization we obtain the thermal properties of neutral particles with a permanent magnetic dipole moment interacting with an external magnetic field. In this way, some new results to the interesting problem are considered in seminal papers by Bakke [13] about the effects of gravitational fields on breaking the infinite degeneracy of the LandauAharonov-Casher levels obtained in flat space-time. The presence of a cosmic string changes the solution as compared with the flat Minkowski space-time results that are due to the combined effects of the curvature and the nontrivial topology determined by the deficit solid angle associated with this space-time. We have investigated the influence of the topological defects on thermal properties. We obtain the solution of the radial part as well as the energy levels. We consider all thermodynamic properties of a neutral particle in a magnetic cosmic string background by using an approach based on the partition function method. We have also analyzed the thermodynamic properties behavior graphically. 
We see that, by increasing the parameters of the topology $\alpha$, the entropy, the heat capacity, and the free energy will also increase. The entropy and the heat capacity are independent of the parameters of the topology. As we expect when $\alpha \rightarrow 1$, we recover the general solution for flat spacetime.

Acknowledgments It is a pleasure for authors to thank the kind referees for their many useful comments on the manuscript.

Open Access This article is distributed under the terms of the Creative Commons Attribution 4.0 International License (http://creativecomm ons.org/licenses/by/4.0/), which permits unrestricted use, distribution, and reproduction in any medium, provided you give appropriate credit to the original author(s) and the source, provide a link to the Creative Commons license, and indicate if changes were made.

Funded by SCOAP ${ }^{3}$.

\section{References}

1. M. Peshkin, A. Tonomura, The Aharonov-Bohm effect, vol. 340. In: Lecture Notes in Physics (Springer, Berlin, 1989)

2. J. Hamilton, Aharonov-Bohm and Other Cyclic Phenomena (Springer, Berlin, 1997)

3. X.G. He, B.H.J. McKellar, Phys. Rev. A 47, 3424 (1993)

4. M. Wilkens, Phys. Rev. Lett. 72, 5 (1994)

5. A. Zeilinger, J. Phys. Colloq. (France) 45, C3-213 (1984)

6. A. Zeilinger, in Fundamental Aspects of Quantum Theory, ed. by V. Gorini, A. Frigero (Plenum, New York, 1985)

7. J. Anandan, Phys. Lett. A 138, 347 (1989)

8. J. Anandan, Phys. Rev. Lett. 85, 1354 (2000)

9. G. Spavieri, Phys. Rev. Lett. 82, 3932 (1999)

10. G. Spavieri, Phys. Rev. A 59, 3194 (1999)

11. G. Spavieri, Phys. Rev. A 59, 3194 (1999)
12. H. Wei, R. Han, X. Wei, Phys. Rev. Lett. 75, 2071 (1995)

13. K. Bakke, Ann. Phys. (Berlin) 524, 338 (2012)

14. K. Bakke, L.R. Ribeiro, C. Furtado, J.R. Nascimento, Phys. Rev. D 79, 024008 (2009)

15. F.M. Andrade, E.O. Silva, M. Pereira, Phys. Rev. D 85, 041701(R) (2012)

16. E.O. Silva, F.M. Andrade, EPL 101, 51005 (2013)

17. M. Ericsson, E. Sjöqvist, Phys. Lett. A 303, 7 (2002)

18. R. Ionicioiu, Phys. Rev. A 68, 034305 (2003)

19. K. Bakke, C. Furtado, Phys. Lett. A 375, 3956 (2011)

20. L.R. Ribeiro et al., Phys. Lett. A 348, 135 (2006)

21. K. Bakke, Int. J. Mod. Phys. A 26, 4239 (2011)

22. Y. Aharonov, D. Bohm, Phys. Rev. 115, 485 (1959)

23. A. Vilenkin, E.P.S. Shellard, Strings and Other Topological Defects (Cambridge University Press, Cambridge, 1994)

24. N.G. Marchuk, Nuovo. Cimento. B 115, 11 (2000)

25. L.D. Landau, E.M. Lifshitz, Quantum Mechanics, Non-relativistic Theory (Pergamon, New York, 1977)

26. G.A. de Marques, V.B. Bezerra, Phys. Rev. D 66, 105011 (2002)

27. R. Bausch, R. Schmitz, L.A. Turski, Phys. Rev. Lett. 80, 2257 (1998)

28. E. Aurell, J. Phys. A: Math. Gen. 32, 571 (1999)

29. K. Bakke, C. Furtado, Phys. Rev. A 80, 032106 (2009)

30. K. Bakke, C. Furtado, Eur. Phys. J. C 69, 531 (2010)

31. C. Furtado, V.B. Bezerrae, F. Moraes, Phys. Lett. A 289, 160 (2001)

32. C. Furtado et al., Phys. Lett. A 195, 90 (1994)

33. K. Bakke, C. Furtado, Phys. Rev. D 80, 024033 (2009)

34. A. Vilenkin, Phys. Rep. 121, 263 (1985)

35. J.S. Dowker, Phys. Rev. D 36, 3095 (1987)

36. V.P. Frolov, E.M. Serebriany, Phys. Rev. D 35, 3779 (1987)

37. T.W.B. Kibble, J. Phys. A 9, 1387 (1976)

38. P. Birrel, P. Davies, Quantum Fields in Curved Space (Cambridge University Press, Cambridge, 1982)

39. H. Kleinert, Gauge Fields in Condensed Matter, vol. 2 (World Scientific, Singapore, 1989)

40. K. Bakke, J.R. Nascimento, C. Furtado, Phys. Rev. D 78, 064012 (2008) 